\title{
Limitations of time resources in human relationships determine social structures
}

Masanori Takano ${ }^{1}$ and Ichiro Fukuda ${ }^{1}$

\begin{abstract}
The number of social relationships that a single human being can possibly be involved in is limited because individuals face time constraints (that is, time costs) in constructing and maintaining social relationships; furthermore, the distribution of the strength of such relationships (as measured by frequency of social interaction) looks significantly skewed (a power law distribution), that is, a few strong relationships and many weak relationships. This skewedness suggests that the costs and benefits of bonding with others depend on the strength of the social relationships: if it involves uniform costs and benefits, the distribution would not be skewed. The bonding is known as social grooming; that is, humans strategically construct their social relationships, and thus, complex human societies should also be strategically constructed. Therefore, it is important to know their strategies for understanding human societies. Previous studies provide evidence of social grooming strategies by examining the evolution and the difference between the various social grooming methods. However, quantitative laws that are important for theoretically understanding human societies are still open to investigation. Social big data is a particularly powerful tool for finding such laws. Therefore, we analyse data from six communication systems (Twitter, a social networking site providing two types of interactions, an avatar chat, a mobile phone and a short message service). We find a simple quantitative law by which social relationships are constrained $\mathrm{Nm}^{a}$ $(a>1)$; here, $N$ is the number of social relationships and $m$ is a mean of the strength of those relationships. The fact that deep social relationships require higher costs per relationship than shallow relationships is suggested by $a>1$ (if the both is equal then $a$ will be 1 ), because the effect of $m$ on the constraint increases with $m$. For exploring why $a$ is greater than 1 , we conduct an individual-based simulation where social grooming costs are assumed to increase linearly with the strength of social relationships. Our results indicate that this model fits all data sets; that is, it displays an explanation capacity for the phenomenon. In addition, an analysis of this simulation proves our assumption about the social grooming cost increasing with the strength of social relationships as being true. Moreover, it suggests that its gradient increases the width and shallowness of these relationships. The law and its causes suggest that mankind's evolution of social grooming has enabled changing social structures, and the phenomenon is because of the constraints of the social network generation. These findings will contribute towards an explanation of the evolution of the various social grooming methods of humans and their significantly large social group.
\end{abstract}

\footnotetext{
${ }^{1}$ Technical Department, CyberAgent, Inc., Tokyo, Japan Correspondence: (e-mail: takano_masanori@cyberagent.co.jp)
} 


\section{Introduction}

$\mathrm{n}$ the basis of the social brain hypothesis (Byrne and Whiten, 1989; Whiten and Byrne, 1997; Dunbar, 2000; Dunbar, 2003), an explanation of social structures, which are typical for humankind, is expected to offer knowledge about human origins, such as the limitation on the number of social relationships (Dunbar, 2000; Dunbar, 2003; Hill and Dunbar, 2003; Gonçalves et al., 2011; Arnaboldi et al., 2013a) and the skewness of social relationships (Zhou et al., 2005; Hossmann et al., 2011; Arnaboldi et al., 2012; Hu et al., 2012; Song et al., 2012; Arnaboldi et al., 2013b; Fujihara and Miwa, 2014), that is, its distributions following a power law (Hossmann et al., 2011; Arnaboldi et al., 2012; Hu et al., 2012; Pachur et al., 2012; Song et al., 2012; Fujihara and Miwa, 2014). These social structures may be caused by human beings' social grooming strategies (Dunbar, 2004).

Social grooming is used to construct and maintain social relationships. This behaviour is important in complex human societies (Dunbar, 2000). Close social relationships lead to mutual cooperation (Uchino et al., 1996; Silk et al., 2003; Hill et al., 2008; Russell and Phelps, 2013; Takano et al., 2016a, b; Dunbar, 2016). A high sociability among baboon mothers, for example, increases the probability of their children's survival, thanks to increased cooperation from others (Silk et al., 2003); similarly, humans tend to cooperate more with close friends than with others (Takano et al., 2016a; Dunbar, 2016). On the other hand, having many weak social relationships helps in obtaining a variety of information which is advantageous in a complex society (Granovetter, 1973; Dunbar, 2004; Eagle et al., 2010; Arnaboldi et al., 2013c).

Social relationships provide humans with various advantages. However, they face cognitive constraints (Dunbar, 2012) (for example, memory and processing capacity) and time constraints (that is, time costs) in constructing and maintaining social relationships. These time costs are not negligible, as humans spend a fifth of their day in social grooming (Dunbar, 1998) and maintaining social relationships (Hill and Dunbar, 2003; Roberts and Dunbar, 2011). Therefore, the mean strength of existing social relationships has a negative correlation with the number of social relationships (Roberts et al., 2009; Miritello et al., 2013a).

Humans must construct and maintain social relationships within the constraints of this trade-off. Thus, we expect that they employ strategies to distribute the limited time resources to maximize benefits from their social relationships (Brown and Brown, 2006; Miritello et al., 2013b; Saramaki et al., 2014). As a result of such strategies, social relationship strengths (as measured by frequency of social grooming (Roberts and Dunbar, 2011; Arnaboldi et al., 2012; Song et al., 2012; Arnaboldi et al., 2013b; Fujihara and Miwa, 2014; Saramaki et al., 2014) may often show a much skewed distribution (Zhou et al., 2005; Arnaboldi et al., 2013b) (distributions following a power law (Hossmann et al., 2011; Arnaboldi et al., 2012; $\mathrm{Hu}$ et al., 2012; Song et al., 2012; Fujihara and Miwa, 2014)).

In other words, human beings' time cost distribution strategies should affect their structures of social relationships. Similarly, the advantage of strategies may depend on social grooming methods because recipients' satisfaction levels depend on social grooming methods, that is, face to face and video call communications show higher satisfaction levels than phone and text communications (Vlahovic et al., 2013), and their time and efforts involved are also different. Humans have various social grooming methods, such as physical contacts (Nelson, 2007), gaze grooming (Kobayashi and Kohshima, 1997; Kobayashi and Hashiya, 2011), gossip (Dunbar, 2004), greetings, phone calls (Vlahovic et al., 2013), e-mails (Vlahovic et al., 2013) and social networking sites (SNS) (Burke and Kraut, 2014; Scissors et al., 2016). These methods will have differing effects in the formation of social relationships and on the time costs.

Human beings maintain large complex social groups by using various and effective social grooming methods efficiently (Dunbar, 2004). Humans have acquired these methods during the evolutionary process. Apes, which are closely related to humans, clean each other's fur as social grooming (Nakamura, 2003). In contrast, humans almost do not do grooming (Nelson, 2007). Alternatively, human beings do lightweight social grooming which has evolved to adapt large groups, for example, gaze grooming (Kobayashi and Kohshima, 1997; Kobayashi and Hashiya, 2011) and gossip (Dunbar, 2004) that enable humans to have several social relationships and less time and efforts are required for these methods. These previous studies provide evidence of social grooming strategies by examining the evolution of social grooming methods. However, quantitative laws that are important for theoretically understanding human societies are still open to investigation. In addition, humans often invest in novel social grooming methods on the Internet, such as social networking sites, online chats and video calls. We will need to know the effects of social grooming methods on social relationships for a safe and comfortable relationship. However, little is known about its effects and mechanisms (Arnaboldi et al., 2013c).

This study aims to discover how social grooming methods influence time cost distribution strategies of the methods and social structures depending on those strategies. We analyse the following six data sets of communication systems: Twitter data (used as a test set in (Cheng et al., 2010), 755 group chats (see the Supplementary information (SI) Fig. 1 for specifications), 755 wall communications (see S1 Fig. for specifications), Japanese avatar chat Ameba Pigg (see Supplementary information Fig. 2 for specifications), mobile phone calls (Madan et al, 2012), and short message service (SMS) (Madan et al, 2012). These human behaviour data sets enable us to analyse social interactions among several users quantitatively. They are useful for constructing theoretical models of social phenomena. Studies (Arnaboldi et al., 2012; Song et al., 2012; Fujihara and Miwa, 2014; Hossmann et al., 2011; Arnaboldi et al., 2013b; Hu et al., 2012; Takano et al., 2016a; Saramaki et al., 2014; Dunbar, 2016; Takano et al., 2016b) previously cited in this paper have also analysed data from SNSs, mobile phones, social network games, and SMSs.

\section{Data analysis}

Data sets. We used six data sets (see Table 1 for details): 1) Twitter data (used as test set in (Cheng et al., 2010) recording interactions among 2,585 people with 278,475 relationships, from 23 June 2007 to 17 March 2010, where an act of social grooming was defined as using the "mention" or "reply" functions to communicate with others; 2) and 3) Data from the Japanese SNS 755, published by 7gogo, Inc. (http://7gogo.jp/), which provides two types of communication systems data, dating from 1 January 2015 to 31 March 2015, which we treated as two different sets (see Supplementary information Fig. 1 for specifications), namely, data from group chats and that from wall communications. The former data records interactions among 17,796 users with 238,611 relationships, where we defined an act of social grooming as communicating in a chat limited to two members. The latter data records interactions among 20,000 users with 534,475 relationships, where we defined an act of social grooming as posting a comment on another's wall. We removed data relevant to official users from both data sets; 4) Data from Japanese avatar chat Ameba Pigg, published by CyberAgent, Inc. (https://pigg.ameba. $\mathrm{jp} /$ ), which records interactions among 76,379 users with $1,610,710$ relationships, from 1 October 2014 to 31 December 
a

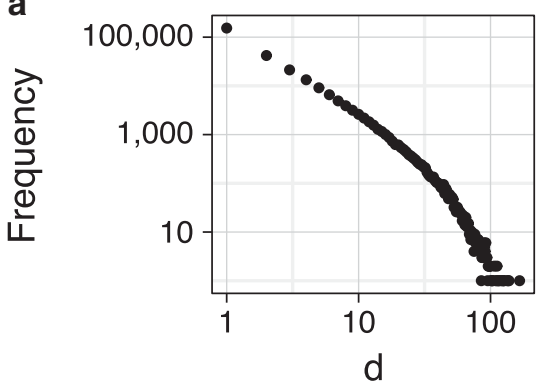

d

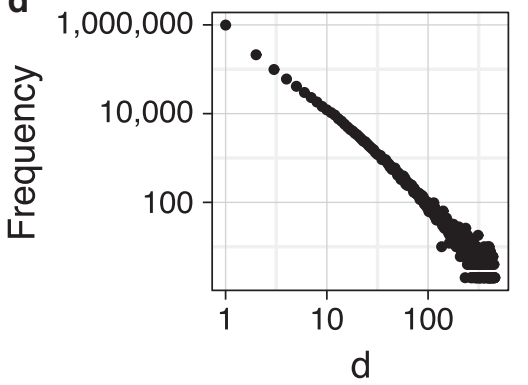

b

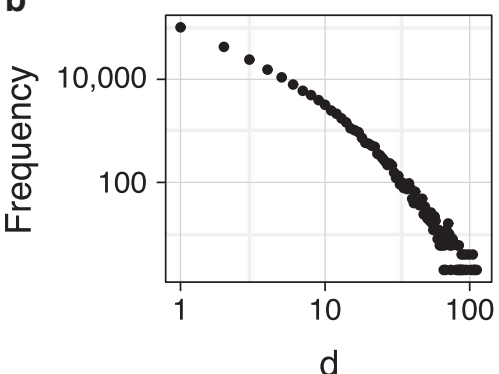

e

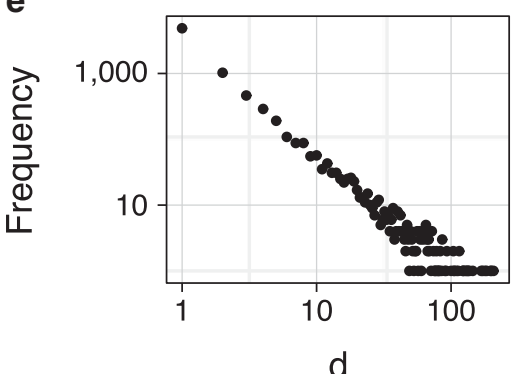

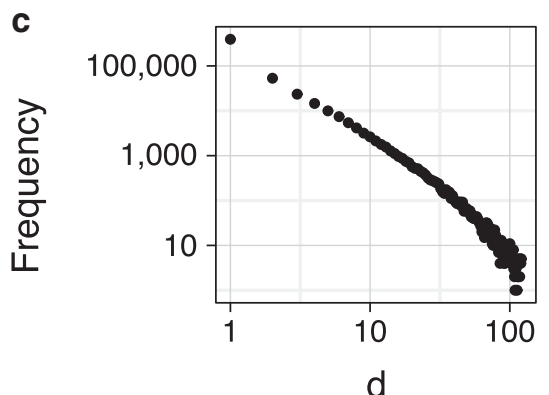

f

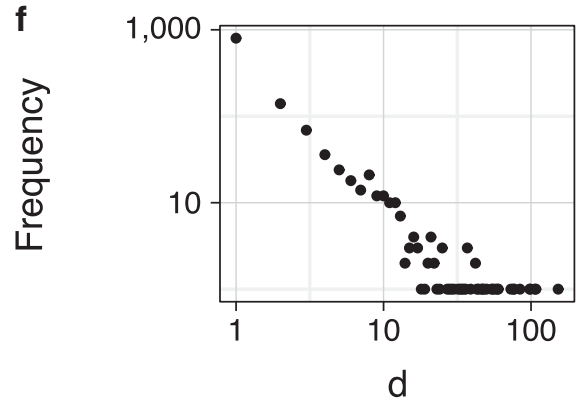

Figure 1 | Power law distributions of number of days $d_{i j}$, where individual $i$ interacted with individual $j$. These results were similar to those of previous studies (Hossmann et al., 2011; Hu et al., 2012; Arnaboldi et al., 2012; Song et al., 2012; Fujihara and Miwa, 2014). Power law coefficients were 1.92 (a: Twitter), 3.71 (b: 755 group chat), 2.29 (c: 755 wall communication), 1.97 (d: Ameba Pigg), 1.98 (e: mobile phone) and 2.00 (f: SMS).
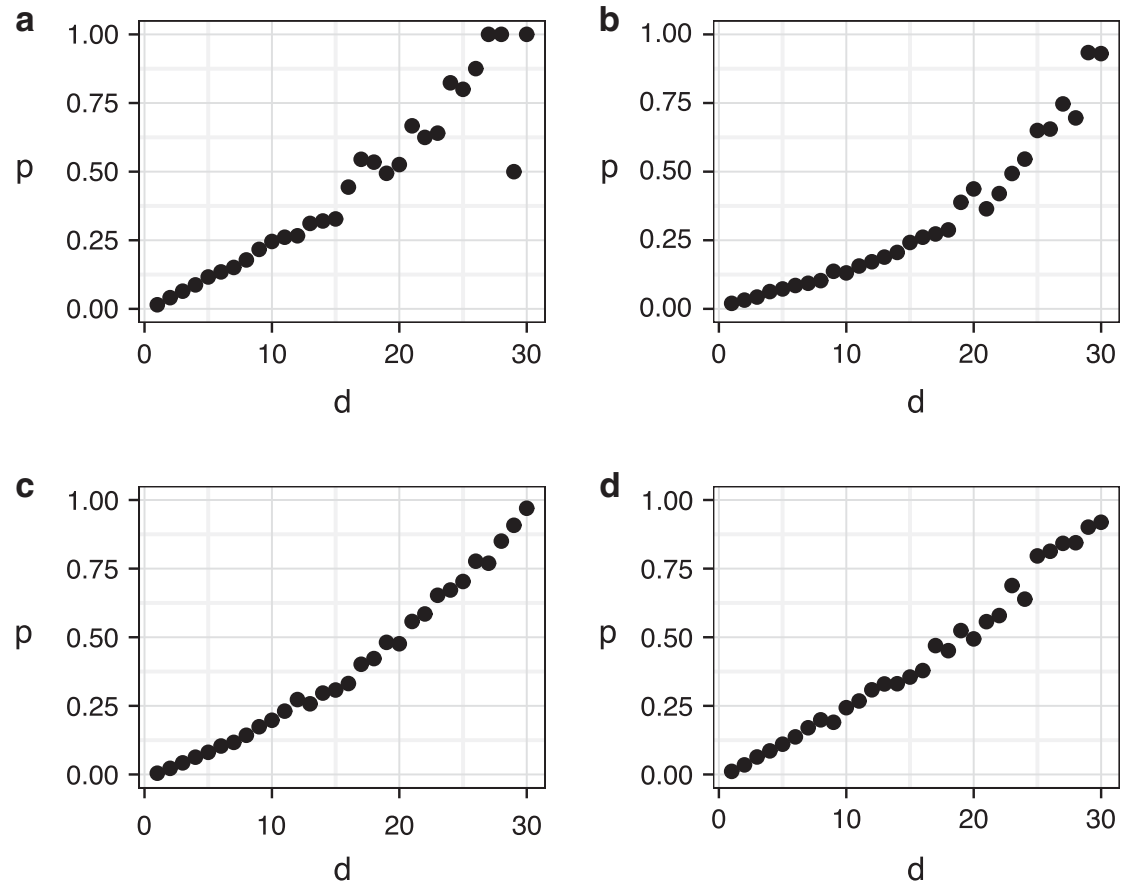

Figure 2 | The Yule-Simon process on social grooming strategies. (a) Twitter, (b) 755 group chat, (c) 755 wall communication, (d) Ameba Pigg. These figures show probability $p$ of social grooming on days after each strength of social relationship $d$. This indicates that the power law distributions were generated by the Yule-Simon process because the $p$ was proportional to $d$, and these strategies subsequently generated the power law distributions. The data periods were from the first 30 days. We did not observe the similar trends in the mobile phone and SMS data sets because of insufficient data. These results were similar in a previous study (Pachur et al., 2012).

2015 (see Supplementary information Fig. 2 for specifications), where we defined an act of social grooming as communicating in a chat limited to two members; 5) Data from mobile phone calls (Madan et al., 2012), recording mobile phone calls among 73 people with 7,805 relationships from 5 September 2008 to 29 June
2009, where we defined an act of social grooming as a call to another; 6) Data from SMS (Madan et al., 2012), which records SMSs among 61 people with 2,266 relationships from 1 January 2008 to 27 June 2009, where we defined an act of social grooming as sending a message to another. 


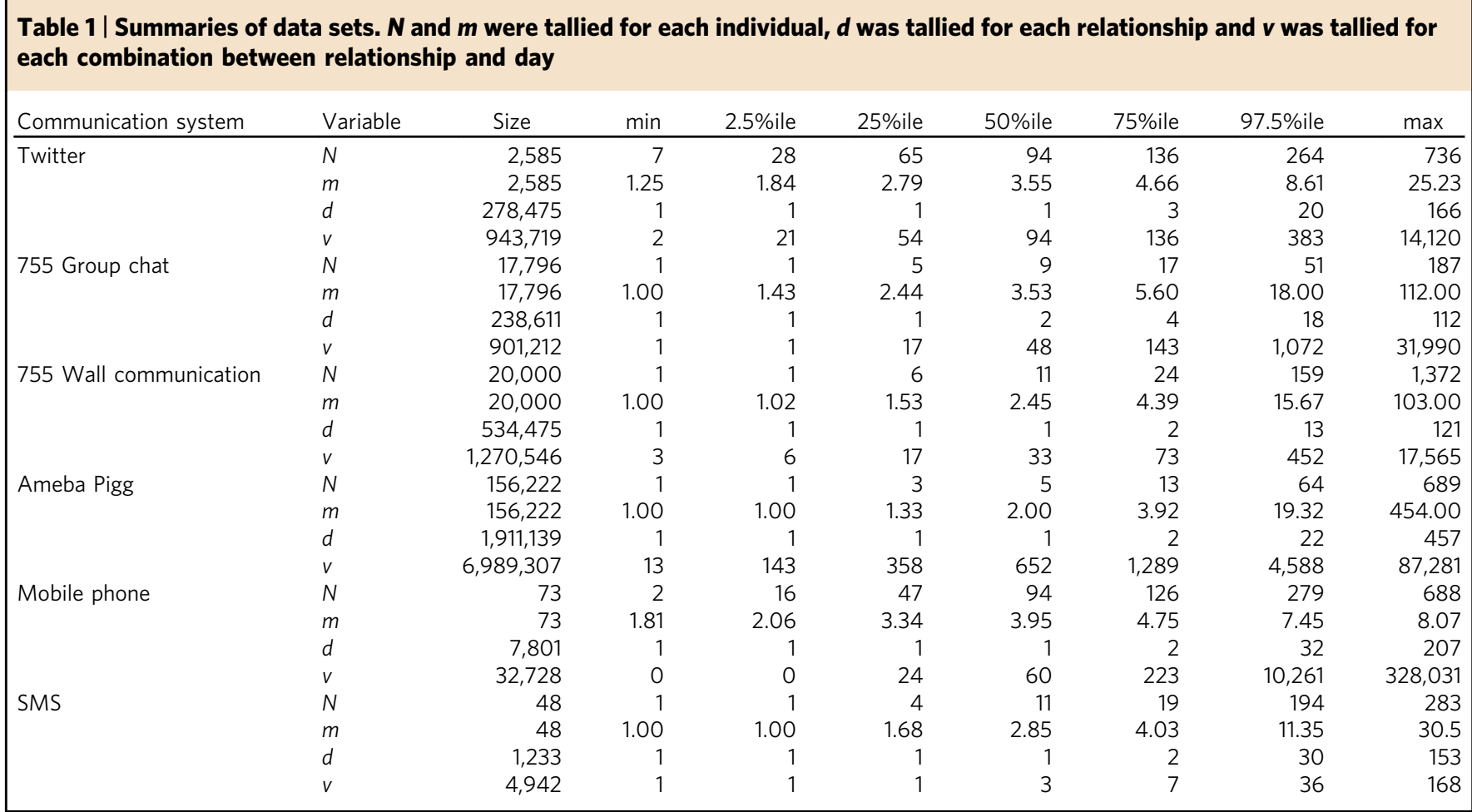

In the data sets from Twitter, 755 (group chat and wall communication) and Ameba Pigg, we limited the targets of analysis to active users who had more number of social grooming days than the 50th percentile among Twitter users and the 75th percentile among 755 and Ameba Pigg users because these internet service data sets included many inactive users. In this article, we defined the strength of social relationships $d_{i j}$ as the days on which individual $i$ does social grooming to individual $j$.

Analysis. In the six communication systems examined in this study, the distributions of the strengths of social relationships $d$ showed power law distributions (Fig. 1), similar to those observed in previous studies (Arnaboldi et al., 2012; Song et al., 2012; Fujihara and Miwa, 2014; Hossmann et al., 2011; Hu et al., 2012). Individuals selected social grooming partners in proportion to the strength of their social relationships, that is, the individuals tended to reinforce their strong social relationships; these power law distributions were generated by the Yule-Simon process (Yule, 1925; Simon, 1955; Newman, 2005) (Fig. 2), similar to a previous study (Pachur et al., 2012). Thus, individuals distributed limited time resources in proportion to the strength of their social relationships and this led to the further strengthening of these relationships. It shows that individuals selected social grooming partners depending on the strength of their social relationships.

As above, these skew distributions seem to be caused by individuals' time cost distribution strategies. Here, we considered a simple model as a null model to analysed how individuals construct social relationships depending on their strength under time cost constraints. If the daily social grooming cost is independent of the strength of the relationship then an individual's total social grooming cost $C$ is $\sum_{i=1}^{N} d_{i}$, where $d_{i}$ is the strength of social relationships from the individual to individual $i$, and $N$ is the total number of social grooming partners. If $m$ is the mean of strengths of social relationships (that is, $\left.\sum_{i=1}^{N} d_{i} / N\right)$, then $C=\sum_{i=1}^{N} d_{i}=N m$. Therefore, under this assumption, $N$ should be inversely proportional to $m$. We conducted the following regression analysis to confirm this hypothesis in the data sets:

$\log N \sim \operatorname{Normal}(\mu, \sigma)$

$\mu=-a \log m+b \log u$,

where $u$ is the number of days of participation for each user, that is, we assumed that a user's total social grooming costs were equal to the number of days for which he/she had participated in the activity $\left(C=u^{b}\right)$. If this hypothesis is correct, then $a$ (the coefficient of $\log m$ ) should be 1 .

Figure 3 shows that all data sets obey $C=N m^{a}(a>1)$ (see Table 2 for the regression results). That is, the null model did not fit the data sets, that is, social grooming cost is not independent of the strength of the relationship. In other words, individuals who had a few strong relationships (that is, large $m$ ) invested more in relationships than individuals who had many weak relationships (that is, small $m$ ). $a>1$ shows that for $C=N m^{a}$, the stronger the social relationship $d$, the more social grooming costs increased per day because the effect of $m$ on $C$ increases with $m$.

To determine how the strength of relationship $d$ affects social grooming costs, we analysed the relation between communication volumes $v$ and the strength of social relationships $d$. Figure 4 shows that the strengths increased the volumes of communication per day, that is, social grooming costs also probably increased with increase in strengths. Figure 5 shows that the increase in gradients was analogized in each data set, that is, this shows gradients depending on social grooming density as distinct from those depending on social grooming frequency. That is, the results of the analysis show that communication volumes $v$ increased along with the relationship strength $d$ and the gradients were independent of the number of days of the data periods $t$. Consequently, social grooming cost should increase with an increase in social grooming density $(d / t)$, under the assumption that social grooming costs are proportional to communication volume $v$. 
a

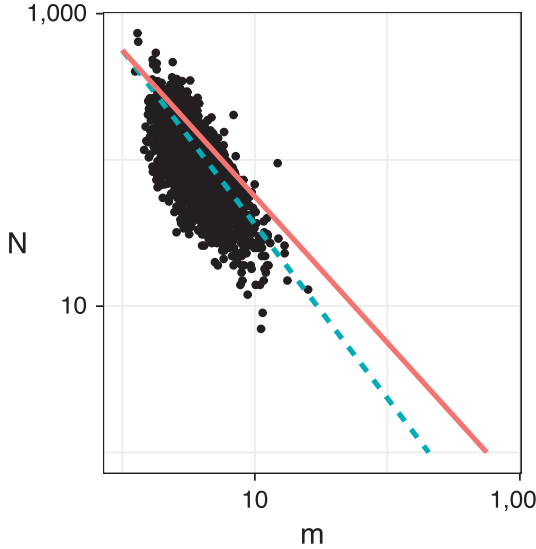

d

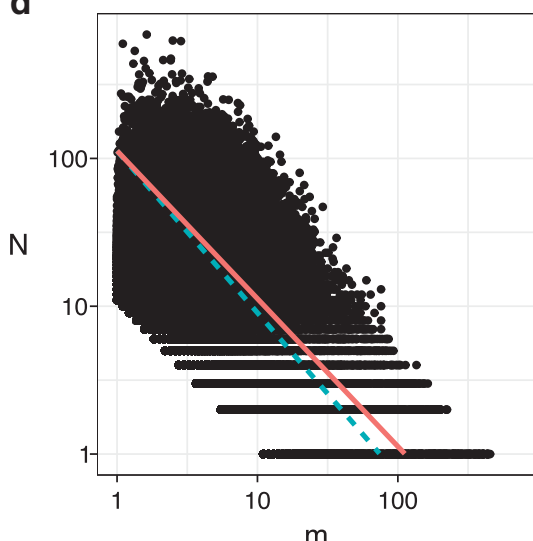

b

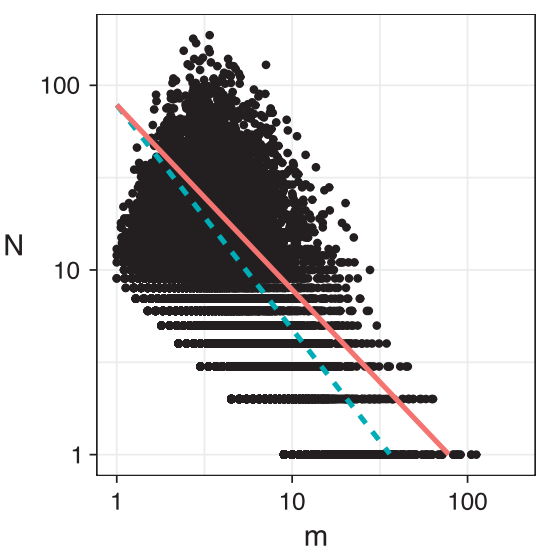

e

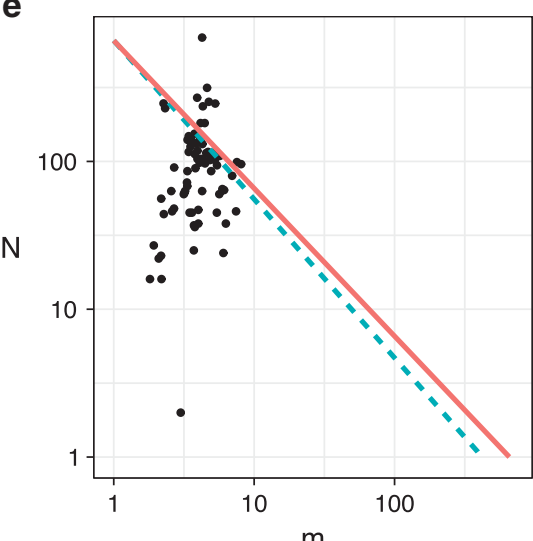

C

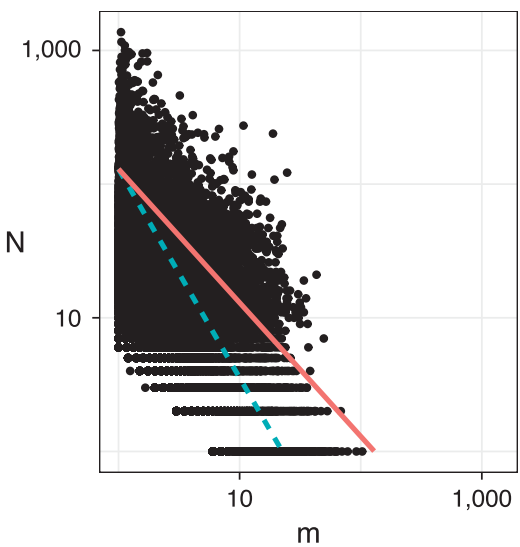

f

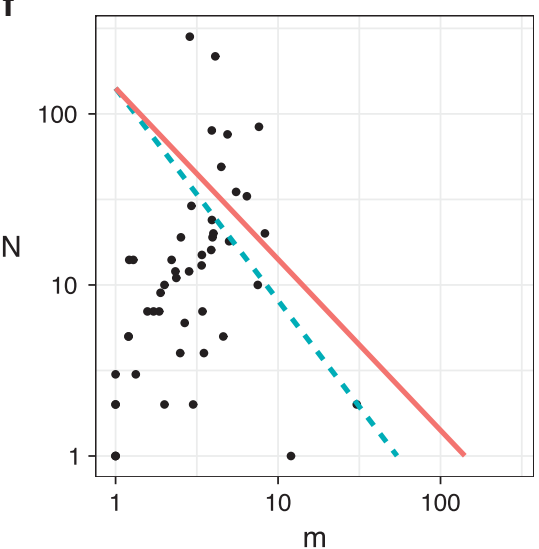

Figure 3 | A simple law between $\boldsymbol{N}$ (total number of social grooming partners) and $\boldsymbol{m}$ (mean number of social grooming days). These user behaviour data (black points) did not obey the null model $(C=N m$; orange lines), where the assumed social grooming cost was independent of the strength of the relationship, but obeyed $C=N m^{a}$ (green and dash lines), where $a=1.19$ (a: Twitter), $a=1.21$ (b: 755 group chat), $a=1.56$ (c: 755 wall communication), $a=1.10$ (d: Ameba Pigg), $a=1.07$ (e: mobile phone) and $a=1.24$ (f: SMS), where user use-days $u$ were 75 th percentile, estimated by the regression models Equation (1), where adjusted R-squares were 0.990 (a: Twitter), 0.974 (b: 755 group chat), 0.959 (c: 755 wall communication), 0.997 (d: Ameba Pigg), 0.994 (e: mobile phone), 0.990 (f: SMS) (see Table 2 for details), and $a>1$ were significant excluding the mobile phone data set.

Table 2 | The results of the regression analysis Equation (1) of each communication system

\begin{tabular}{llllll} 
Communication System & Coefficient & Estimate & Standard Error & t-value & P-value \\
\hline Twitter & $a$ & 1.189567 & 0.023256 & 8.15 & $4.4 \times 10^{-16}$ \\
& $b$ & 1.309346 & 0.006815 & 192.12 & Less than $2.0 \times 10^{-16}$ \\
755 Group chat & $a$ & 1.214229 & 0.004640 & 46.17 & Less than $2.0 \times 10^{-16}$ \\
& $b$ & 1.269766 & 0.002294 & 553.5 & Less than $2.0 \times 10^{-16}$ \\
755 Wall communication & $a$ & 1.562142 & 0.006250 & 89.94 & Less than $2.0 \times 10^{-16}$ \\
& $b$ & 1.476393 & 0.002769 & 533.2 & Less than $2.0 \times 10^{-16}$ \\
Ameba Pigg & $a$ & 1.0954104 & 0.0007440 & 128.24 & Less than 2.0 \\
& $b$ & 1.0939529 & 0.0003137 & 3487 & Less than $2.0 \times 10^{-16}$ \\
Mobile phone & $a$ & 1.07332 & 0.15756 & 0.47 & $3.2 \times 10^{-1}$ \\
SMS & $b$ & 1.25628 & 0.04689 & 26.795 & Less than $2.0 \times 10^{-16}$ \\
& $a$ & 1.24089 & 0.07815 & 3.08 & $3.5 \times 10^{-3}$ \\
& $b$ & 1.21949 & 0.02995 & 40.72 & Less than 2.0
\end{tabular}

The $t$-values and the $p$-values of $a$ measuring the statistical uncertainty in coefficient $a$ are larger than 1 . The $t$-values and the $p$-values of $b$ measuring the statistical uncertainty in coefficient $b$ are not equal to 0 . The coefficient $a$ was larger than 1 , that is, the user behaviour data did not obey the null model $(C=N m ; a=1)$. Their adjusted $R^{2}$ values were 0.990 (Twitter), 0.974 ( 755 group chat), 0.959 (755 wall communication), 0.997 (Ameba Pigg), 0.994 (mobile phone) and 0.990 (SMS)

\section{Individual-based simulation}

Model. To explain $a>1$ for $C=N m^{a}$, we constructed a simulation model of social grooming cost distribution strategies based on the assumption that social grooming costs increase with an increase in social grooming density $d / t$. We used a linear social grooming cost function $c(d)=\alpha d / t+\beta$ as the simplest assumption (if $\alpha=0$ then it is the null model). In the model, we considered two type individuals, which were groomers and groomees, and groomers construct social relationships using their limited resources (that is, time), based on this assumption and the Yule-Simon process. 
a
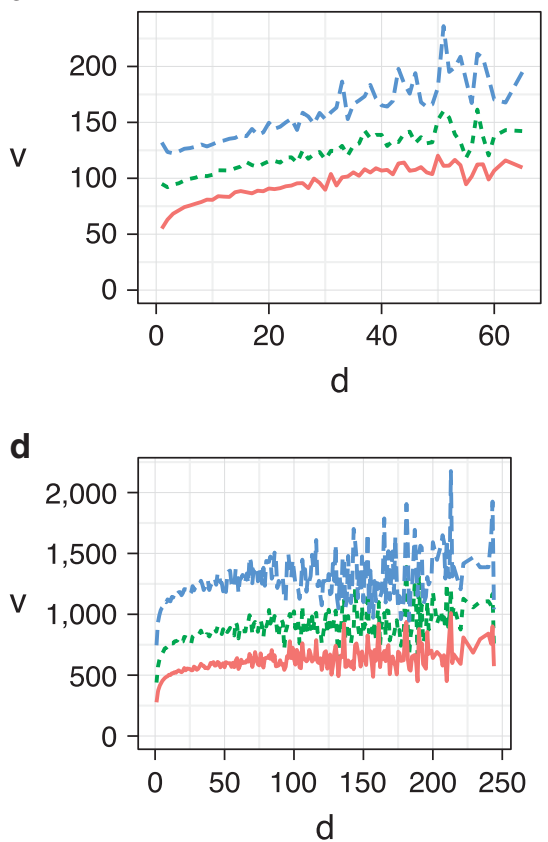

b

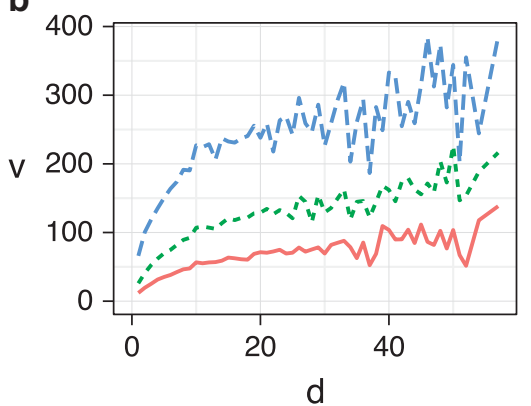

e

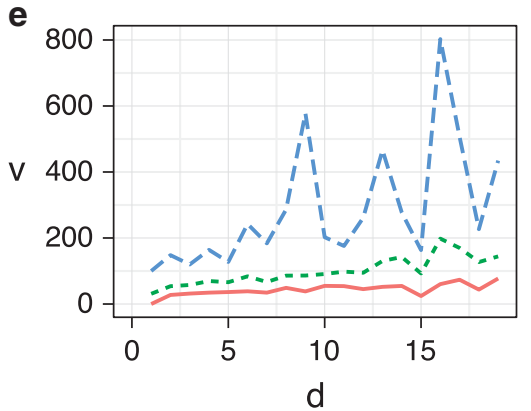

C

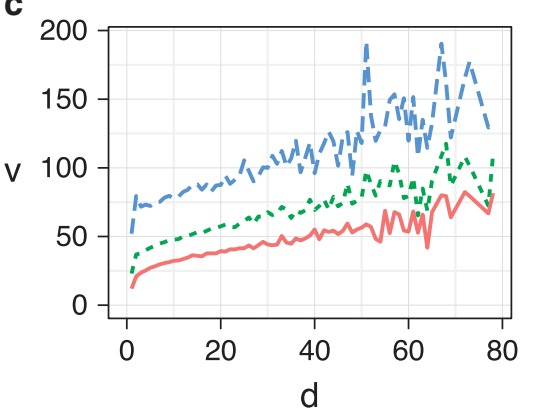

f

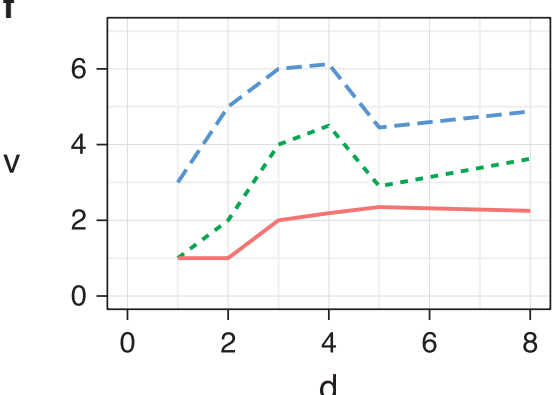

Figure 4 | Increasing communication volumes per day $\mathbf{v}$ by strengths of social relationships $d$, where the volumes were number of characters per day in Twitter, 755 and Ameba Pigg, duration per day in mobile phones and frequency of messaging per day in SMS because we did not consider any information regarding number of characters in the SMS data set. The orange lines are the 25th percentile, the green and dotted lines are the 50th percentile and the blue and dashed lines are the 75th percentile. These are shown for cases where the number of samples was more than 20 (the ranges of the $d$ of mobile phone and SMS are short because these were smaller data sets). (a) Twitter, (b) 755 group chat, (c) 755 wall communication, (d) Ameba Pigg, (e) Mobile phone, (f) SMS.

a

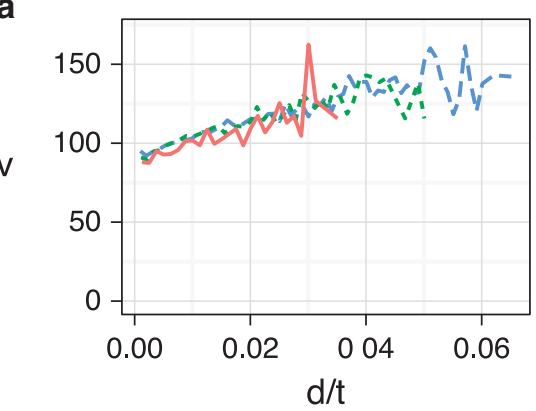

d

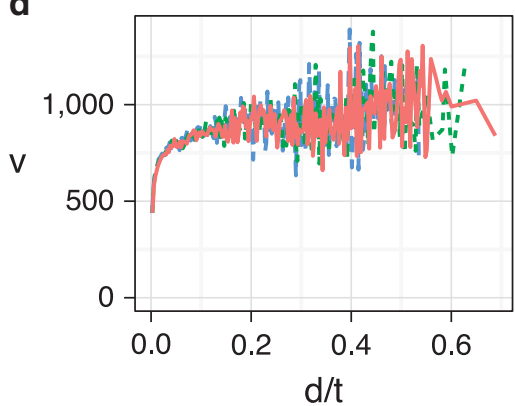

b

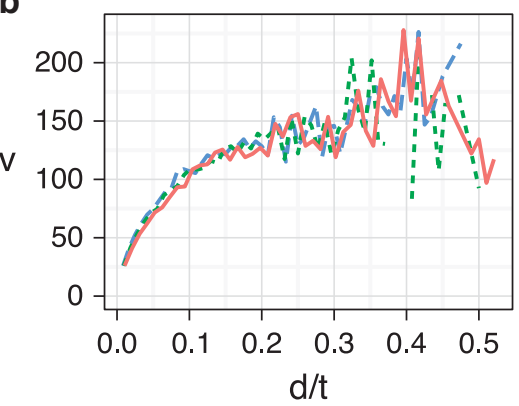

e

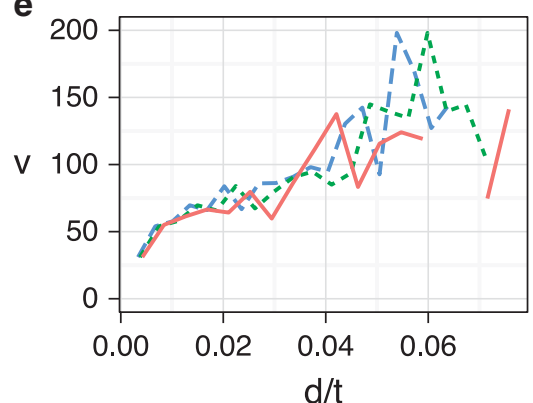

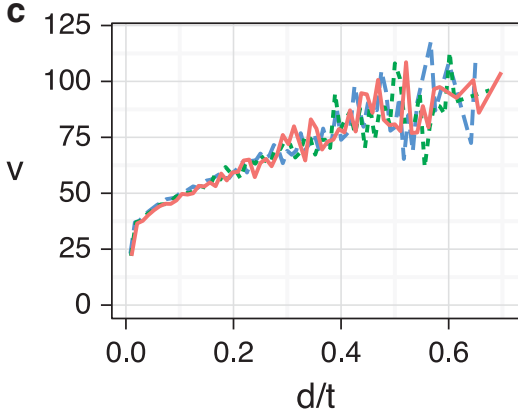

f

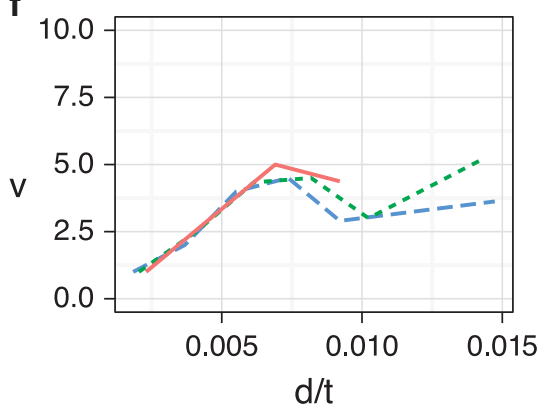

Figure 5 | The gradients of communication volumes depending on social grooming density as distinct from those depending on social grooming frequency. (a) Twitter, (b) 755 group chat, (c) 755 wall communication, (d) Ameba Pigg, (e) Mobile phone, (f) SMS. These figures show compaction of the medians of $v$ for each social grooming density $(d / t)$ for different periods ( $t$ is number of days of the data periods). Each line represents entire periods (orange lines), nine-tenths of the periods (green and dotted lines), and eight-tenths of the periods (blue and dashed lines). These are shown when the number of samples is more than $\mathbf{2 0}$ (the ranges of $d$ of mobile phone and SMS are short because the sizes of these data sets were small).

We conducted the following simulation for $T$ step to construct social relationships $d_{i j}$. At each step $t$, groomer $i$ repeats the following for $R>0 . R$ is reset to an initial value $R_{0}$ before each step $t$. Each $i$ has a resource $R$ that is spent when $i$ performs social grooming with others. Each $i$ creates a social relationship with a stranger, groomee $j$, depending on probability $q_{i}$, where the strength of social relationship $d_{i j}$ is 1 and $i$ pays the cost $\beta$ from its resource $R$ (if $R<\beta$, then $d_{i j}$ is $R / \beta$ and $R$ becomes 0 ). In contrast, 
a

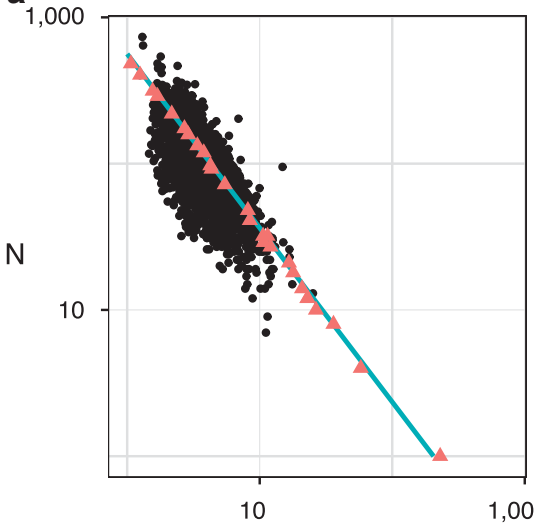

$\mathrm{m}$

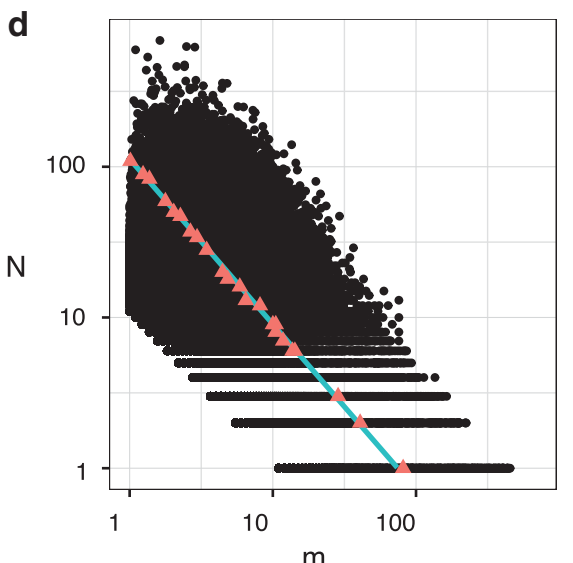

b

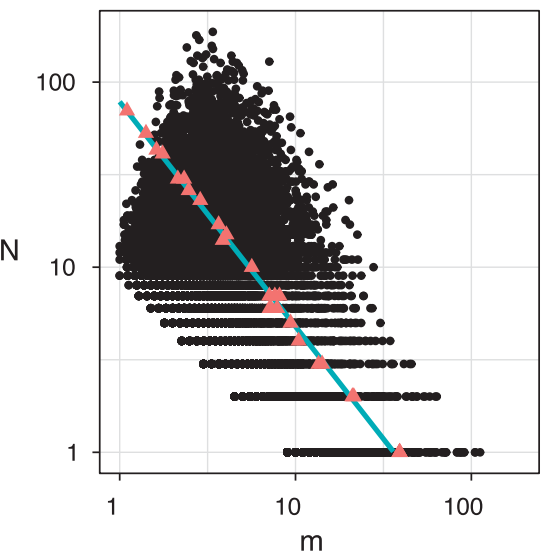

e

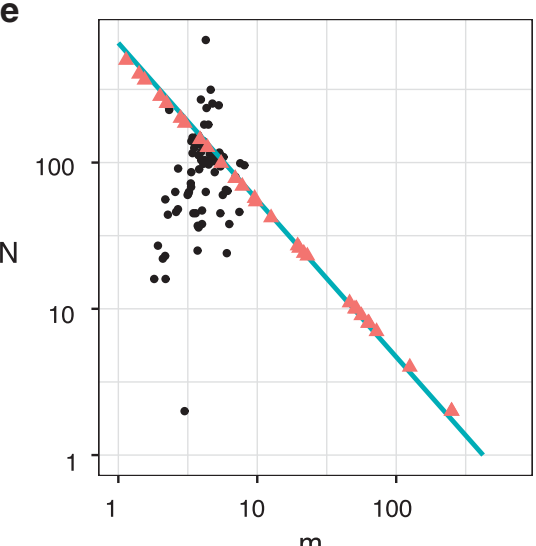

C

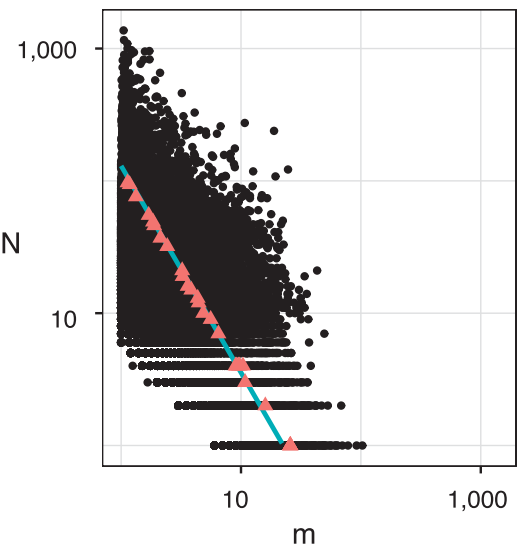

$\mathbf{f}$

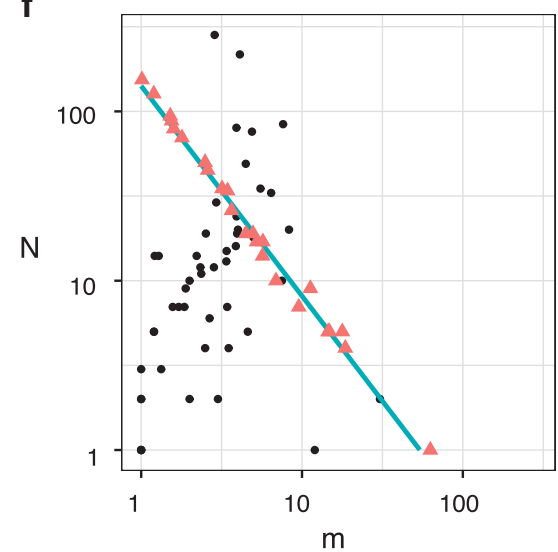

Figure 6 | Explanation capacity of the simulation model. The results of fits by the simulation model to the regression lines of all data sets (that is, green and dashed lines in Fig. 3). Very good fits were observed between the simulation results (orange triangles) and the regression lines (green lines); that is, the simulation model displayed explanation capacity for the phenomenon $a>1$ for $C=N m^{a}$, with a monotonically increasing cost function ( $\alpha>0$ ). The parameters of the cost functions were $\alpha=1.34$ and $\beta=0.24$ (a: Twitter), $\alpha=1.27$ and $\beta=0.39$ (b: 755 group chat), $\alpha=3.89$ and $\beta=0.23$ (c: 755 wall communication), $\alpha=1.62$ and $\beta=0.64$ (d: Ameba Pigg), $\alpha=0.05$ and $\beta=0.31$ (e: mobile phone) and $\alpha=5.05$ and $\beta=0.34$ (f: SMS).

$i$ reinforces its social relationships depending on probability $1-q_{i}$. Each $i$ selects a social grooming partner $j$ depending on a probability proportional to the strength of the social relationships between $i$ and $j\left(d_{i j}\right)$, then $i$ adds 1 to the strength of its social relationship (that is, the Yule-Simon process) and pays the cost $c$ $\left(d_{i j}\right)=\alpha d_{i j} / t+\beta$ from $R$ (if $R<c(d)$, then $i$ adds $R / c(d)$ to its strength of social relationship $d_{i j}$ and $R$ becomes 0 ). Each $i$ does not perform the act of social grooming twice with the same groomees in each step $t$. Therefore, selected groomees are excluded from the selection process of a social grooming partner $j$ in each step $t$.

\section{Experiments}

First, we tested the fit of this model. That is, we optimized parameters $\alpha$ and $\beta$ to fit the regression lines of Fig. 3, where the evaluation function was the mean square error between simulation results and regression lines, $T$ was the period for each data set, $R_{0}$ was the 75 th percentile of each user's use-days $u$ divided by $T\left(R_{0}=0.126\right.$ and $T=998$ (Twitter), $R_{0}=0.258$ and $T=120$ (755 group chats), $R_{0}=0.225$ and $T=120$ (755 wall communications), $R_{0}=0.164$ and $T=456$ (Ameba Pigg), $R_{0}=0.589$ and $T=297$ (mobile phone) and $R_{0}=0.107$ and $T=543$ (SMS)). The number of groomees was set large enough. If our hypothesis is correct then $\alpha>0$. We found that this model fitted all data sets (Fig. 6), that is, it had an explanation capacity for the phenomenon $a>1$ for $C=N m^{a}$, with a monotonically increasing cost function $(\alpha>0)$. It demonstrates that the phenomenon $a>1$ can be attributed to a groomers' tendency to invest higher costs in strong rather than weak social relationships.

Next, we analysed the effect of parameter $\alpha$ on the structure of social relationships by using the model; we used $T, R$ and $\beta$ of the Twitter data set, as in the former experiment. As the result demonstrates, $a$ was determined by the cost function gradient $\alpha$ (Fig. 7a), and the power law coefficients of the strength of social relationships increased with the gradient $\alpha$ (Figs. 7b and 7c), that is, the gradient increased the width and shallowness of social relationships. Because the increase of the gradient $\alpha$ decreased the number of social relationships of groomers with strong social relationships, they have to invest time in strong social relationships to maintain these relationships. Consequently, increase in $\alpha$ decreased strong social relationships, and structures of social relationships became relatively wide and shallow.

\section{Discussion}

There is a trade-off between the number of social relationships (that is, $N$ ) and the mean strength of social relationships (that is, m) (Roberts et al., 2009; Miritello et al., 2013a) as humans must perform frequent social grooming to maintain close relationships 
a

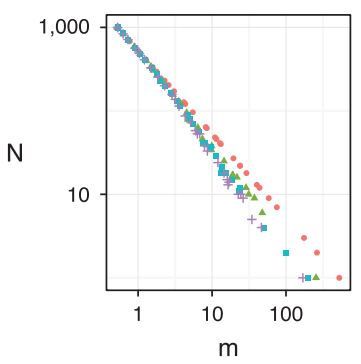

b
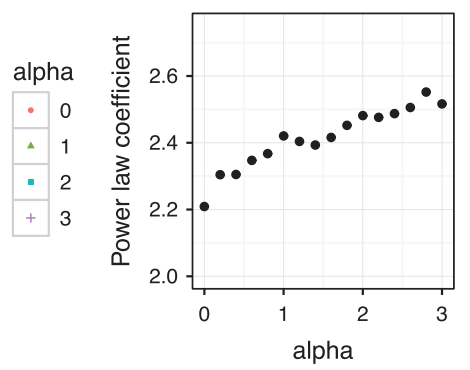

C

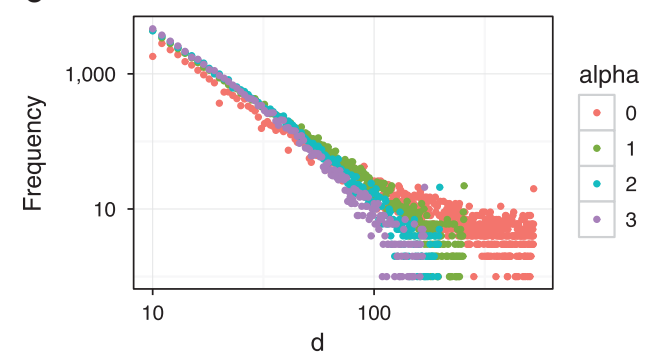

Figure 7 | Effect of the gradient of grooming cost function $\alpha$ on $\mathbf{C}=\mathrm{Nm}^{a}$ and the distribution of the strengths of social relationships in the simulation. (a) Simulation result instances $\left(C=N m^{a}\right)$, (b) The effect of $\alpha$ on the power law coefficients of the distributions of social relationships strengths, (c) Simulation result instances (the distributions of social relationships strengths). Figure a shows that gradient $\alpha$ increased $a$. Figures $\mathrm{b}$ and $\mathrm{c}$ show that the gradient $\alpha$ increased the power law coefficients of the strengths of social relationships, that is, the gradient increased width and shallowness of social relationships. The parameters $T, R$ and $\beta$ were based on Twitter (see Fig. 6a) because $\beta$ has no effect on $a$. In figures $a$ and $b$, the number of individuals was 200 and $q_{i}$ included the 200 values used in $[0,1]$. In figure $c$, the number of individuals was 10,000 and $q_{i}$ included the 10,000 values used in $[0,1]$.

(Hill and Dunbar, 2003; Roberts and Dunbar, 2011; Saramaki et al., 2014). Here, we found a simple law where $N$ was inversely proportional to $m^{a}(a>1)$. The $a$ is because of the increase in social grooming costs; the costs increase with the strengths of social relationships. This cost increase may be because of the fact that strong social relationships tend to be the site of complex and frequent communications.

We also found that the gradient of the cost increase was an important factor to determine the structure of social relationships. In communication systems with the large gradient of social grooming costs, people tend to construct wide and shallow social relationships. In contrast, people tend to maintain close social relationships with limited partners in the communication system with a small gradient of social grooming costs.

The variation of the gradient may explain why human beings use different social grooming methods according to their different social relationships. Human beings' lightweight social grooming has evolved to adapt large groups, for example, gaze grooming (Kobayashi and Kohshima, 1997; Kobayashi and Hashiya, 2011) and gossip (Dunbar, 2004), because these grooming methods enable humans to have several social relationships and require less time and efforts. For example, in text communications over the Internet, such as on Twitter, users tend to construct wide and shallow social relationships that are used for acquiring and diffusing information (Arnaboldi et al., 2013c; Preoiuc-Pietro et al., 2015). Thus, lightweight social grooming is effective in constructing many weak social relationships. In contrast, elaborate social grooming methods are more effective than lightweight social grooming methods in reinforcing social relationships (Burke and Kraut, 2014). In addition, for strong social relationships, human beings prefer elaborate social grooming methods, such as face-to-face or telephone communications, rather than text communications over the Internet (Burke and Kraut, 2014). It may be because the degree of satisfaction from elaborate social grooming methods (face to face and video calls) tend to be higher than that from lightweight social grooming methods (phone and text communication) (Vlahovic et al., 2013). Therefore, we infer that the gradient of elaborate social grooming costs is smaller than that of lightweight social grooming costs, and the intercept of lightweight social grooming methods is smaller than that of elaborate social grooming methods. Thereby, human beings tend to construct new social relationships and maintain weak social relationships by using lightweight social grooming methods, and they tend to use elaborate social grooming methods to maintain strong social relationships. It thus suggests that an emergence of novel social grooming methods can change social structures, for example, the evolution of gaze grooming (Kobayashi and Kohshima, 1997; Kobayashi and Hashiya, 2011) and gossip (Dunbar, 2004). That is, the evolution of human beings' lightweight social grooming to adapt large groups suggests that they have made wide and shallow societies. It may shed light on the emergence of our huge modern societies. In addition, our findings also suggest predictability of the invention effects of novel communications over the Internet on social structures, that is, in the novel lightweight social grooming will drive wide and shallow social structures, and novel elaborate social grooming will drive narrow and deep social structures.

The strengthening of already strong social relationships (the Yule-Simon process which generates power law distributions) and the positive gradient of social grooming costs, which represent a time cost distribution strategy, seem to have been caused by competing for cooperating with others. That is, strong social relationships may exist for receiving cooperation from others (Brown and Brown, 2006; Miritello et al., 2013b). However, cooperators cannot cooperate with everyone because there are costs of cooperation (Santos et al., 2006; Xu and Wang, 2015). Actually, human beings tend to cooperate with close friends (Haan et al., 2006; Harrison et al., 2011; Dunbar, 2016). Consequently, human beings would need to compete based on the strength of the social relationships with the cooperators' friends and this may generate a skewed distribution for the strength of social relationships. Exploring evolutionary stability of this strategy for cooperation will provide knowledge about evolutionary dynamics of human significant social intelligence based on social brain hypothesis.

Additionally, our findings provide novel insight on social network sciences, that is, a constraint on the construction of social relationships $\left(C=\mathrm{Nm}^{a}\right)$ and its effect on social network structures, for example, they create constraints for the dynamics of network generation and temporal networks.

\section{References}

Arnaboldi V, Conti M, Passarella A and Dunbar RIM (2013c) Dynamics of personal social relationships in online social networks: a study on Twitter. In: Proceedings of the first ACM conference on Online social networks, ACM Press: New York, pp 15-26.

Arnaboldi V, Conti M, Passarella A and Pezzoni F (2012) Analysis of ego network structure in online social networks. In: 2012 International Conference on Privacy, Security, Risk and Trust and 2012 International Confernece on Social Computing, Washington, DC, pp 31-40.

Arnaboldi V, Conti M, Passarella A and Pezzoni F (2013b) Ego networks in Twitter: an experimental analysis. In: International Conference on Computer Communications, Turin, Italy, pp 3459-3464.

Arnaboldi V, Guazzini A and Passarella A (2013a) Egocentric online social networks: analysis of key features and prediction of tie strength in Facebook. Computer Communications; 36 (10-11): 1130-1144. 
Brown SL and Brown RM (2006) Selective investment theory: recasting the functional significance of close relationships. Psychological Inquiry; 17 (1): 1-29.

Burke M and Kraut RE (2014) Growing closer on facebook: changes in tie strength through social network site use. In: Proceedings of the 32nd annual ACM conference on Human factors in computing systems, ACM Press: New York, pp 4187-4196.

Byrne R and Whiten A (1989) Machiavellian Intelligence: Social Expertise and the Evolution of Intellect in Monkeys, Apes, and Humans. Oxford University Press: Oxford.

Cheng Z, Caverlee J and Lee K (2010) You are where you tweet: a content-based approach to geo-locating twitter users. In: Proceedings of the 19th ACM international conference on Information and knowledge management, ACM Press: New York, pp 759.

Dunbar RIM (1998) Theory of mind and the evolution of language. In: Hurford JR, Studdert-Kennedy M and Knight C (eds). Approaches to the Evolution of Language: Social and Cognitive Bases. Cambridge University Press: Cambridge, UK, pp 92-110.

Dunbar RIM (2000) On the origin of the human mind. In: Carruthers P and Chamberlain A (eds). Evolution and the Human Mind. Cambridge University Press: Cambridge, UK, pp 238-253.

Dunbar RIM (2003) The social brain: Mind, language, and society in evolutionary perspective. Annual Review of Anthropology; 32 (1): 163-181.

Dunbar RIM (2004) Gossip in evolutionary perspective. Review of General Psychology; 8 (2): 100-110.

Dunbar RIM (2012) Social cognition on the internet: testing constraints on social network size. Philosophical transactions of the Royal Society of London. Series B, Biological sciences; 367 (1599): 2192-2201.

Dunbar RIM (2016) Do online social media cut through the constraints that limit the size of offline social networks? Royal Society Open Science; 3, 150292.

Eagle N, Macy M and Claxton R (2010) Network diversity and economic development. Science; 328 (5981): 1029-1031.

Fujihara A and Miwa H (2014) Homesick Lévy Walk: A mobility model having Ichi-Go Ichi-e and scale-free properties of human encounters. In: IEEE Annual Computer Software and Applications Conference, Västerås, Sweden, pp 576-583.

Gonçalves B, Perra N and Vespignani A (2011) Modeling users' activity on twitter networks: validation of Dunbar's number. PLoS ONE; 6 (8): e22656.

Granovetter M (1973) The strength of weak ties. American Journal of Sociology; 78 (1): $1360-1380$

Haan M, Kooreman P and Riemersma T (2006) Friendship in a public good experiment. IZA Discussion Paper; 2108.

Harrison F, Sciberras J and James R (2011) Strength of social tie predicts cooperative investment in a human social network. PLoS ONE; 6 (3): e18338.

Hill RA, Bentley RA and Dunbar RIM (2008) Network scaling reveals consistent fractal pattern in hierarchical mammalian societies. Biology Letters; 4 (6): 748-751.

Hill RA and Dunbar RIM (2003) Social network size in humans. Human Nature; 14 (1): $53-72$.

Hossmann T, Spyropoulos T and Legendre F (2011) A complex network analysis of human mobility. In: IEEE Conference on Computer Communications Workshops, Shanghai, China, pp 876-881.

Hu T, Wenning BL, Görg C, Toseef U and Guo Z (2012) Statistical analysis of contact patterns between human-carried mobile devices. In: 4th International Conference on Mobile Networks and Management, pp 244-257.

Kobayashi H and Hashiya K (2011) The gaze that grooms: contribution of social factors to the evolution of primate eye morphology. Evolution and Human Behavior; 32 (3): 157-165.

Kobayashi H and Kohshima S (1997) Unique morphology of the human eye. Nature; 387 (6635): 767-768.

Madan A, Cebrian M, Moturu S, Farrahi K and Pentland AS (2012) Sensing the "health state" of a community. IEEE Pervasive Computing; 11 (4): 36-45.

Miritello G et al (2013a) Time as a limited resource: communication strategy in mobile phone networks. Social Networks; 35 (1): 89-95.

Miritello G, Lara R, Cebrian M and Moro E (2013b) Limited communication capacity unveils strategies for human interaction. Scientific Reports; 3, 1950.

Nakamura M (2003) 'Gatherings' of social grooming among wild chimpanzees: implications for evolution of sociality. Journal of Human Evolution; 44 (1): 59-71.

Nelson H (2007) Encoding and decoding mutual grooming: Communication with a specialized form of touch In: Dissertation Abstracts International, SectionB: The Sciences and Engineering. 68.

Newman MEJ (2005) Power laws, Pareto distributions and Zipf's law. Contemporary Physics; 46, pp 323-351.

Pachur T, Schooler LJ and Stevens JR (2012) When will we meet again? Regularities of social connectivity and their reflections in memory and decision making. In: Hertwig R, Hoffrage U and ABC Research Group (eds). Simple Heuristics in a Social World. Oxford University Press: Oxford, pp 199-224.

Preoiuc-Pietro D, Volkova S, Lampos V, Bachrach Y and Aletras N (2015) Studying User Income through Language, Behaviour and Affect in Social Media. PLoS ONE; 10 (9): e0138717.

Roberts SGB and Dunbar RIM (2011) Communication in social networks: Effects of kinship, network size, and emotional closeness. Personal Relationships; 18 (3): 439-452.
Roberts SGB, Dunbar RIM, Pollet TV and Kuppens T (2009) Exploring variation in active network size: Constraints and ego characteristics. Social Networks; 31 (2): $138-146$.

Russell YI and Phelps S (2013) How do you measure pleasure? A discussion about intrinsic costs and benefits in primate allogrooming. Biology \& Philosophy; 28 (6): 1005-1020.

Santos FC, Rodrigues JF and Pacheco JM (2006) Graph topology plays a determinant role in the evolution of cooperation. Proceedings of the Royal Society B: Biological Sciences; 273 (1582): 51-55.

Saramaki J, Leicht EA, Lópezb E, Roberts SGB, Reed-Tsochas F and Dunbar RIM (2014) Persistence of social signatures in human communication. Proceedings of the National Academy of Sciences; 111 (3): 942-947.

Scissors L, Burke M and Wengrovitz S (2016) What's in a Like? Attitudes and behaviors around receiving Likes on Facebook. In: Proceedings of the 19th ACM Conference on Computer-Supported Cooperative Work \& Social Computing, ACM Press: New York, pp 1499-1508.

Silk JB, Alberts SC and Altmann J (2003) Social bonds of female baboons enhance infant survival. Science; 302 (5648): 1231-1234.

Simon HA (1955) On a class of skew distribution functions. Biometrika; 42 (3-4): $425-440$.

Song C, Wang D and Barabasi AL (2012) Connections between human dynamics and network science, http://arxiv.org/abs/1209.1411, accessed 8 February 2017

Takano M (2016) Communication volumes in social relationships in online communication systems. Figshare. https://doi.org/10.6084/m9.figshare.3395956.v3.

Takano M, Wada K and Fukuda I (2016a) Reciprocal altruism-based cooperation in a social network game. New Generation Computing; 34 (3): 257-271.

Takano M, Wada K and Fukuda I (2016b) Lightweight Interactions for Reciprocal Cooperation in a Social Network Game. In: Social Informatics: 8th International Conference, Springer International Publishing: Bellevue, WA, pp 125-137.

Uchino BN, Cacioppo JT and Kiecolt-Glaser JK (1996) The relationship between social support and physiological processes: a review with emphasis on underlying mechanisms and implications for health. Psychological Bulletin; 119 (3): 488-531.

Vlahovic T, Roberts SGB and Dunbar RIM (2013) "Media Naturalness Theory" and Human Social Bonding. In: Human Behavior and Evolution Society Conference, Miami Beach, FL.

Whiten A and Byrne R (1997) Machiavellian Intelligence II: Extensions and Evaluations. Cambridge University Press: Cambridge, UK.

$\mathrm{Xu} \mathrm{B}$ and Wang J (2015) The emergence of relationship-based cooperation. Scientific Reports; 5, 16447.

Yule GU (1925) A mathematical theory of evolution, based on the conclusions of Dr J. C. Willis, F.R.S. Philosophical Transactions of the Royal Society B: Biological Sciences; 213 (402-410): 21-87.

Zhou WX, Sornette D, Hill RA and Dunbar RIM (2005) Discrete hierarchical organization of social group sizes. Proceedings of the Royal Society B: Biological Sciences; 272 (1561): 439-444.

\section{Data availability}

All data needed to evaluate the conclusions in the article are present in the article, the Supplementary information, and the dataset deposited in Figshare (Takano 2016: https:// doi.org/10.6084/m9.figshare.3395956.v3).

\section{Acknowledgements}

The authors are grateful to Assistant Professor Genki Ichinose at Shizuoka University and $\mathrm{Mr}$ Yoshihito Hotta at University of Tokyo, for their valuable comments and suggestions throughout this study.

\section{Additional information}

Supplementary Information: accompanies this paper at http://www.palgrave-journals .com/palcomms

Competing interests: The authors declare no competing financial interests.

Reprints and permission information is available at http://www.palgrave-journals.com/ pal/authors/rights_and_permissions.html

How to cite this article: Takano M and Fukuda I (2017) Limitations of time resources in human relationships determine social structures. Palgrave Communications. 3:17014 doi 10.1057/palcomms.2017.14

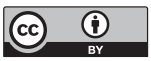

This work is licensed under a Creative Commons Attribution 4.0 International License. The images or other third party material in this article are included in the article's Creative Commons license, unless indicated otherwise in the credit line; if the material is not included under the Creative Commons license, users will need to obtain permission from the license holder to reproduce the material. To view a copy of this license, visit http://creativecommons.org/licenses/by/4.0/ 